\title{
Brightness of the corona with the height according to observations of SOHO/EIT during 1996-2003
}

\author{
A. G. Tlatov ${ }^{1}$ and V. I. Makarov ${ }^{2}$ \\ ${ }^{1}$ Kislovodsk Solar Station of the Pulkovo Observatory, Russia, email: solar@narzan.ru \\ ${ }^{2}$ Pulkovo Observatory, St.Petersburg, Russia, email: makarov@gao.spb.ru
}

\begin{abstract}
An analysis of the distribution of coronal brightness in the UV radiation according to SOHO/EIT in the wavelengths $171 \AA$, $195 \AA$ and $304 \AA$ was carried out. A change of coronal brightness at the heights $1.02 R-1.20 R$ radius of the Sun was considered during 1996-2003. The distribution of brightness of the corona at the various heights depends on the phase of the solar cycle. Brightness of the corona at the heights more $1.05 \mathrm{R}$ in the wavelengths $171 \AA$ and $195 \AA$ has the greatest brightness during an epoch of the maximum activity. Brightness of the internal corona at the heights less $1.05 R$ has the lowered brightness during this period. The maximum intensity of the corona $304 \AA$ was observed in the maximum activity at all heights. Comparison brightness of the corona with the number bright points in UV was carried out. For this purpose the bright points in the wavelength $304 \AA$ were allocated on the solar disk of the daily observations. The number of the bright points has the maximum on the phase of the minimum activity while the area of the bright regions has maximum in the maximum activity.
\end{abstract}

The initial data were daily observations on the telescope SOHO/EIT in the wavelengths $171 \AA, 195 \AA$ and $304 \AA$. For determination of the corona brightness we have to determine the brightness calibration of the images. Here we used the standard calibration procedure of the images (Benevolenskaya, Kosovichev \& Scherrer(2001). As a result of this procedure the new rows have been generated.

The corona intensity measured at some height $h$ above limb of the Sun brings the information on brightness of the corona directly on the height $h$ and about overlying layers. It is well known that the corona is formed in the layer of small optical thickness. Then the flux of radiation $S_{h}$ measured at the height $h$ is proportional to the integral from intensity along the beam of the sight $I_{h}$, or for all heights above $h: S_{h} \sim \int I_{r} d r$. Integration is carried out from $r=h$ up to some height where brightness of the corona in this spectral range can be accepted the zero. As a first approximation for definition of intensity of the corona $I_{h}$ at the height $h$ can use numerical differentiation $I_{h} \sim$ $\left(S_{r_{1}}-S_{r_{2}}\right) /\left(r_{1}-r_{2}\right)$. Here $S_{r_{1}}$ and $S_{r_{2}}$ are the flux of the radiation from the corona measured at the heights $r_{1}$ and $r_{2}$ close to the height h. Further we shall interest the relative behaviour of the corona brightness at the given height in the cycle. Therefore the constant factors here were excluded. The brightness $I_{h}$ was calculated as a difference of intensity $\left(S_{r_{1}}-S_{r_{2}}\right)$ at $d r=\left(r_{1}-r_{2}\right)=0.01 R$.

On the Figures 1 the latitude-time diagrams of the corona intensity for the wavelengths $171 \AA$ at the heights $r=1.02 R, 1.05 R, 1.10 R$ are presented. The brightness of the corona depends on the wavelength and the height. The corona brightness in the $171 \AA$ and $195 \AA$ has appreciably various behavior on the low $r<1.05 \cdot R$ and high $r>1.05 \cdot R$ heights. At the middle and low latitude of the Sun brightness of the high corona has the typical structure of the butterflies, connected with the sunspot activity. Brightness of the low corona has maximum on the phase of the minimum and decrease of the sunspot activity. 

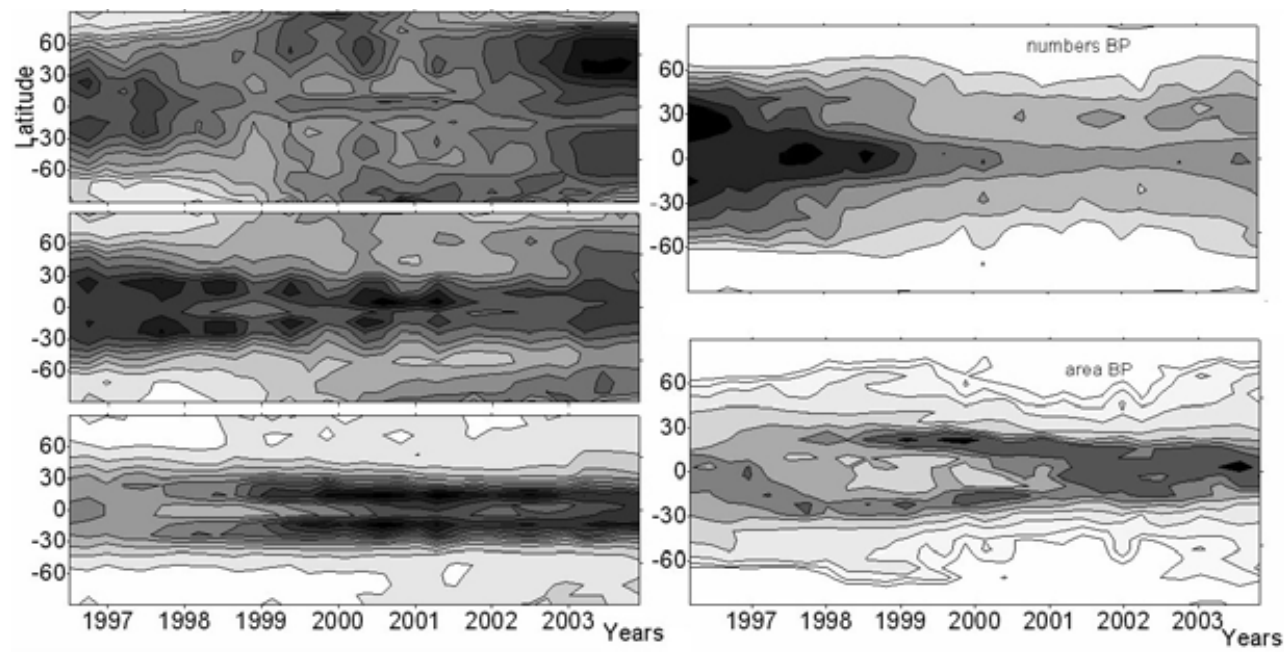

Figure 1. Brightness of the corona at the heights $r=1.02 R, 1.05 R, 1.10 R$ according to the observations SOHO/EIT-171 ̊ during 1996-2003.

Figure 2. The latitude-time diagram of the number of the bright points allocated on the solar disk in the line EIT-304A (the top panel) and the areas of the bright elements (the bottom panel).

On the phase of the maximum activity the low corona at the middle latitude has low brightness. The behavior of the corona at the wavelength $304 \AA$ has identical character at the various heights.

Different behavior of the low and high corona (brightness) during the solar cycle 23 testifies on the different mechanisms of formation of the corona with the height. The high corona $(r>1.05 R)$ is formed, on the whole, from the arch structures. The high of the arches are determined the properties of the bipolar magnetic structures. In the maximum cycle these arches are raised enough high. The nature of the corona brightness on these high shows that the local maximums in the different hemispheres are formed simultaneously and they re-cover the equator. The diffusion corona, probably, is formed by other mechanism. The nature of the corona brightness shows, that this type of the corona is formed under action of the small-scale component of the magnetic field. These regions are made of the local sources of the small size of the mixed polarity.

This assumption we testify by the allocation of the bright points on the SOHO/EIT$304 \AA$ images. For determination of the bright points we have to select the level on which their border is emerged. For this purpose the function of distribution of the element brightness on the calibrated images was calculated. The Figure 2 shows the latitude time diagrams for the number (the top panel) and for the area of the bright elements (the bottom panel). As things turned out, the number of the bright points has maximum in the minimum activity while the area of the bright structures has maximum on the phase of the maximum activity.

\section{References}

Benevolenskaya, E.A., Kosovichev, A.G. \& Sherer, P.H. 2001 Ap.J. 554, 107L. 